Document downloaded from:

http://hdl.handle.net/10251/63863

This paper must be cited as:

M. Winston Caldwell; Gómez Barquero, D.; Caldwell, MW. (2015). Broadcast television spectrum incentive auctions in the U.S.: trends, challenges, and opportunities. IEEE Communications Magazine. 53(7):50-56. doi:10.1109/MCOM.2015.7158265.

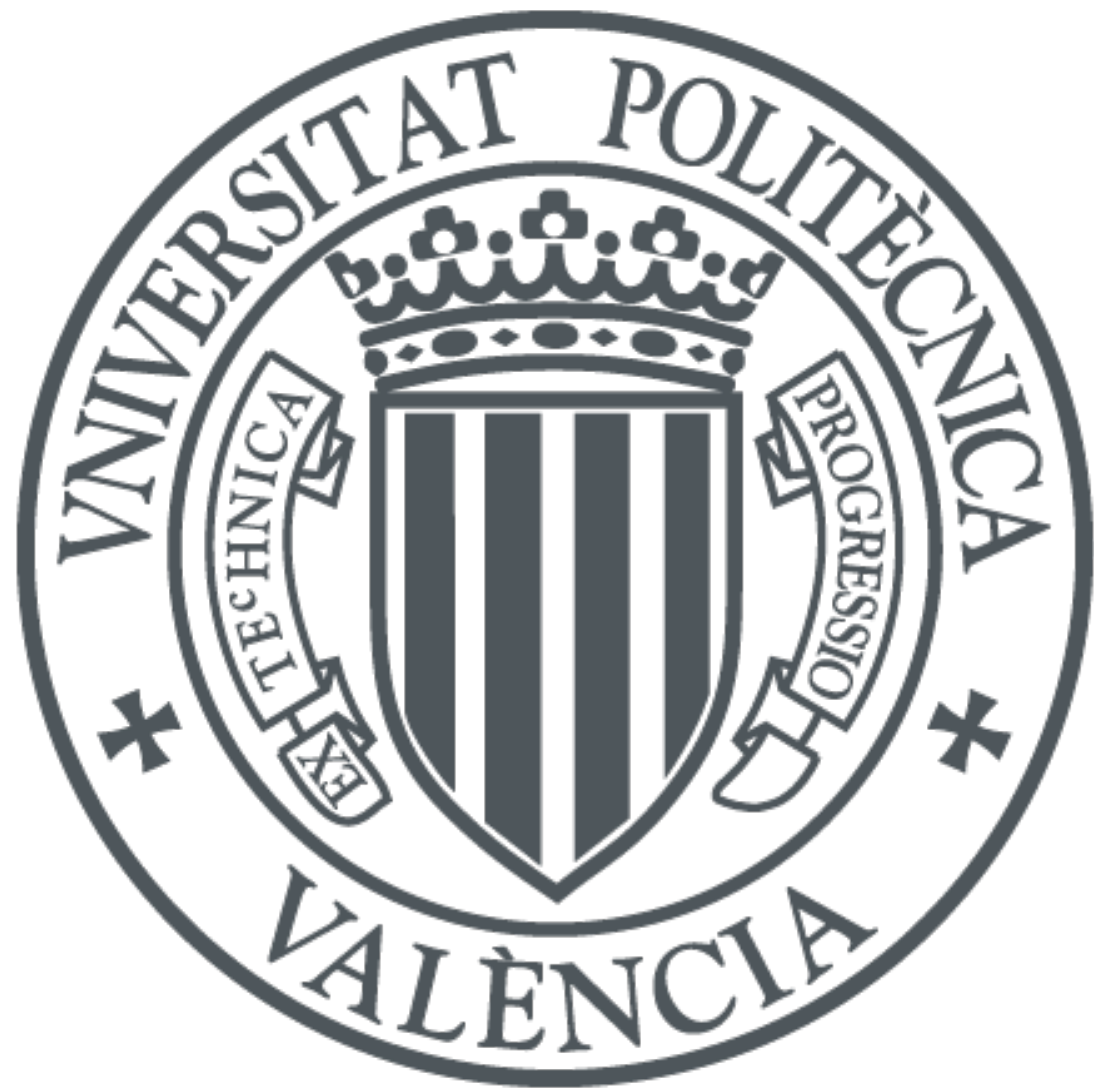

The final publication is available at

http://dx.doi.org/10.1109/MCOM.2015.7158265

Copyright Institute of Electrical and Electronics Engineers (IEEE)

Additional Information

(C) (c) 2015 IEEE. Personal use of this material is permitted. Permission from IEEE must be obtained for all other uses, in any current or future media, including reprinting/republishing this material for advertising or promotional purposes, creating new collective works, for resale or redistribution to servers or lists, or reuse of any copyrighted component of this work in other works. 


\title{
Broadcast Television Spectrum Incentive Auctions in the U.S.: Trends, Challenges, and Opportunities
}

\begin{abstract}
This paper presents an overview of the upcoming television broadcast spectrum incentive auction in the U.S., which will be the first ever attempted worldwide, and discusses the main business, regulatory and technical challenges of a successful incentive auction. The process combines two separate but linked auctions: a reverse auction, which will identify the prices at which broadcasters are willing to relinquish their spectrum; and a forward auction, which will determine the price mobile network operators are willing to pay to acquire the new frequencies. The two auctions will determine the buyers and sellers and also the amount of spectrum to be cleared in the $600 \mathrm{MHz}$ band after reorganizing the television stations that remain on air. This process is known as repacking and will create contiguous blocks of cleared spectrum at the high frequency side of the UHF band for mobile use. The paper also reviews the potential plans for the $600 \mathrm{MHz}$ band and discusses the opportunities that could bring about the new digital terrestrial television standard known as "ATSC 3.0".
\end{abstract}

\section{KEYWORKS}

4G, 5G, $600 \mathrm{MHz}$ band, ATSC 3.0, Digital Terrestrial Television Broadcasting, FCC, Incentive Auctions, Regulation, Spectrum, UHF, WRC.

\section{INTRODUCTION}

The radio frequency $(\mathrm{RF})$ spectrum is a finite natural resource with considerable economic and social importance. The Ultra-High Frequency (UHF) band from 470 to $862 \mathrm{MHz}$ has traditionally been used for terrestrial television broadcasting [1]. The International Telecommunication Union (ITU) allocated the upper part of the terrestrial broadcasting UHF band to International Mobile Telecommunications (IMT) technologies during the World Radiocommunications Conferences (WRC) of 2007. This band ranges from 790 to $862 \mathrm{MHz}$ in Region 1 (800 MHz band), and from 698 to $790 \mathrm{MHz}$ in Region 2 and Region 3 (700 MHz band), see Figure 1.

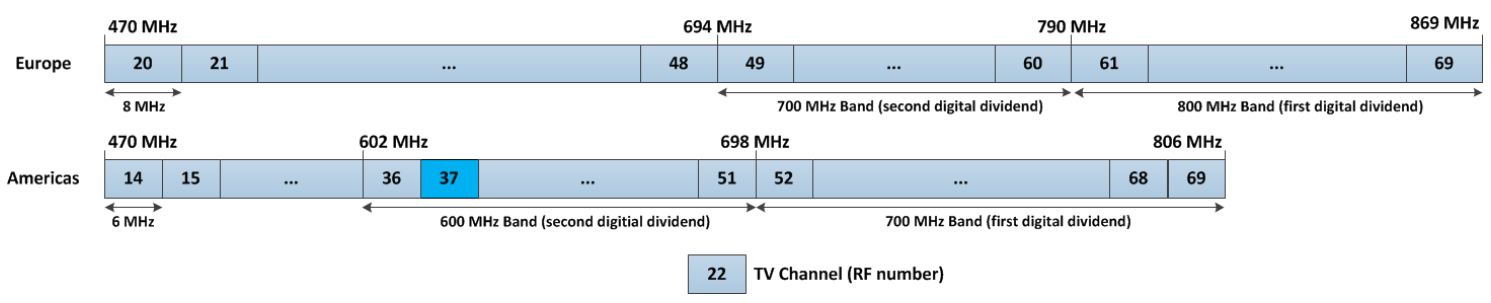

Figure 1: Frequency allocations in the UHF band for digital terrestrial broadcasting in ITU Region 1 (Europe, Middle East and Africa, and Russia) and Region 2 (Americas).

The term digital dividend was introduced to name the television spectrum released during the switch-over from analogue to Digital Terrestrial Television (DTT). Initially, the number of available broadcast TV channels in Europe and the U.S. was 49 and 55 channels, which were reduced down to 40 and 37 after the first digital dividend. After the second digital band in the 
$700 \mathrm{MHz}$ band, only 28 will be left in Europe, whereas in the U.S. it will depend on the outcome of the incentive auction.

Before the analogue TV switch-off took place, most spectrum regulators worldwide auctioned and awarded the digital dividend band to fourth-generation (4G) Long Term Evolution (LTE) mobile networks together with other frequency bands (e.g., 1.8 or $2.6 \mathrm{GHz}$ ). The U.S. was the first country to deploy nation-wide 4G LTE networks in the digital dividend band. The deployments in Europe were initially hampered due to the lack of harmonized spectrum in the region, delays in the analogue TV switch-off, and the popularity of terrestrial television in some countries where DTT is the main television distribution platform. Despite the issues of the deployment of $4 \mathrm{G}$ networks in the digital dividend band, many networks are currently on air or are being deployed, including the Asia-Pacific region. Discussions have turned to the feasibility of a second digital dividend in the terrestrial broadcasting UHF band.

At the WRC-2012, it was agreed to allow for the introduction of mobile broadband services in the $700 \mathrm{MHz}$ band in ITU Regions 1 and 3, to be effective after the upcoming WRC-2015. National administrations in those countries will have the possibility to enable a second digital dividend in that band. Some European countries such as Finland, Germany, Sweden and UK have already announced their intentions to release these frequencies for $4 \mathrm{G}$ LTE (some countries as early as 2017). Moreover, the European Commission (EC) is considering reallocating the $700 \mathrm{MHz}$ band around 2020 [2]. The European Conference of Postal and Telecommunications Administrations (CEPT) has proposed that the established terrestrial broadcasting UHF spectrum below $700 \mathrm{MHz}$ would continue to be used for terrestrial broadcasting and would remain in place until at least 2030.

The $700 \mathrm{MHz}$ band is already being used by mobile networks in the U.S. as the first digital dividend band. The U.S. is taking a further step by proposing the $600 \mathrm{MHz}$ band as a second digital dividend. The Federal Communications Commission (FCC) has introduced a novel market-based spectrum auction scheme known as broadcast television spectrum incentive auction [4], in which broadcasters may voluntarily relinquish their spectrum licenses in exchange for a share of the auction proceeds paid by the Mobile Network Operators (MNOs).

Broadcasters choosing to participate in the auction would have different options depending on their current RF channel assignment, which involve different business and strategic trade-offs. The incentive auction may represent a financial opportunity for broadcasters who remain on the air through the channel sharing option. Doing so, broadcasters would continue their business through a channel sharing agreement and they would not lose must-carry rights over cable and satellite. Depending on the agreement, channel sharing may lower both operating and capital expenditures for the broadcasters involved. To participate in the incentive auction, broadcasters will be offered the possibility to: (i) relinquish its current RF channel and share an RF channel with another broadcaster, (ii) move from the UHF to the VHF band, (iii) move from high VHF to low VHF, or (iv) go off the air.

The process combines two separate but linked auctions: a reverse auction, which will identify the prices at which broadcasters are willing to relinquish their RF channels; and a forward auction, which will determine the price MNOs are willing to pay to acquire the new frequencies. The two auctions will determine the buyers and sellers and also the amount of spectrum to be cleared in the $600 \mathrm{MHz}$ band after reorganizing the television (TV) stations that remain on air. This process is known as repacking and will create contiguous blocks of cleared spectrum at the high frequency side of the UHF band for mobile use.

This paper presents an overview of the upcoming TV broadcast spectrum incentive auction in the U.S. and reviews the main business, regulatory and technical challenges of a successful 
incentive auction. The paper presents the potential plans for the $600 \mathrm{MHz}$ band, which could repurpose up to $144 \mathrm{MHz}$ of broadcast TV spectrum and allocate $120 \mathrm{MHz}$ for mobile broadband. The paper discusses the broadcast frequency repacking and the opportunities that may bring a migration to the new and improved global DTT standard that is currently in development, known as ATSC 3.0.

\section{InCENTIVE AUCTIONS OVERVIEW}

Incentive auctions were introduced in the 2010 U.S. National Broadband Plan [3]. After a few delays, the auction may take place in early 2016, although it should be pointed out that at the day of writing this article the FCC continues to work on determining the final auction rules and procedures. There are several outstanding issues left to be resolved, such as the determination of the initial broadcast spectrum clearing target, opening bid prices, benchmarks for the final stage rule, and the final TV channel assignment process.

Incentive auctions are a market-driven tool for repurposing spectrum. The idea behind the incentive auction is that broadcasters may be willing to voluntarily relinquish all or some of their spectrum usage rights during the reverse auction in exchange for a share of the income raised in the forward auction. Broadcasters may, of course, elect not to participate in the incentive auction. Current estimates, while varying widely, indicate that about 10.7 million TV households in the U.S. rely on DTT only, which represents an approximately $10 \%$ penetration, although the percentage of households with at least one DTT set is considerably larger.

In order for the incentive auction to be carried through to completion, it is required to raise substantial proceeds. Different estimates based on previous spectrum auctions predict that the proceeds could approach up to USD \$45 billion [3]. Compensations may need to be significant for broadcasters not only in the top markets, but also in mid to smaller-sized markets in order to clear spectrum on a nationwide basis.

The incentive auction is structured in two separate but interdependent auctions, known as reverse and forward auctions, linked by the repacking process. For a given spectrum clear target defined by the FCC:

- The reverse auction will determine the price at which broadcasters will voluntarily relinquish their spectrum usage rights and the amount of spectrum available in each market.

- The forward auction will determine the price MNOs are willing to pay for such spectrum.

The repacking process consists of reorganizing and assigning RF channels to the broadcast TV stations that remain on air after the incentive auction in order to create contiguous blocks of cleared spectrum at the upper frequency range of the $600 \mathrm{MHz}$ band for mobile broadband use.

The participation of the broadcasters in the incentive auction is totally flexible and voluntary, and broadcasters that participate have also the possibility to drop out from the auction (these broadcasters will be treated as if they had not participated in the auction). The FCC has devised a flexible scheme to encourage the participation of the broadcasters with four different options, which involve different-business and strategic trade-offs, depending on their current RF channel assignment.

1. Bid to relinquish a UHF channel to move to a high VHF (174 to $216 \mathrm{MHz}$ ) or to a low VHF (54 to $88 \mathrm{MHz}$ ) channel.

2. Bid to relinquish a high VHF channel to move to a low VHF channel. 
3. Bid to relinquish their current UHF channel and share a channel with another broadcaster after the auction.

4. Bid to relinquish their license and go off the air.

Only broadcasters who relinquish their spectrum usage rights will share the auction proceeds based on the value of their spectrum. Broadcasters not relinquishing their spectrum licenses will continue operating in the same frequency band, be subject to a potentially substantial disruptive RF channel reassignment resulting from the repacking process, and be entitled to reimbursement for relocation costs from a USD $\$ 1.75$ billion TV broadcaster relocation fund, which must be generated from the proceeds of the forward auction. It is intended that funds would cover up to $80 \%$ of the eligible costs for commercial operators and up to $90 \%$ for non-commercial stations. Broadcasters that continue over-the-air operation after the auction will retain the mandatory carriage rights on cable and satellite systems.

Broadcasters sharing an RF channel must retain the capability to transmit at least one Standard Definition (SD) TV channel. This is the only constraint imposed by the FCC, and the actual terms of the sharing agreement are left to the broadcasters. Channel sharing agreements should be executed prior to the auction.

Regarding the possibility of migrating from the UHF down to the VHF bands, broadcasters will be able to choose whether to bid for the high- or the low-VHF band, but the actual RF channel will be assigned in the repacking process if the broadcaster's bid is selected in the auction.

Broadcasters relinquishing their license or accepting a channel sharing bid would have to vacate their RF channels within 3 months after receiving the economic compensation. Broadcasters remaining on the air would have up to 39 months after the auction ends to move to the new RF channel assigned in the repacking process.

The reverse and forward auctions will be integrated in a series of stages with a reserve price mechanism such that market forces will determine the amount of cleared spectrum and the revenues raised. Each stage will consist of a reverse auction followed by a forward auction for a given spectrum clear target defined by the FCC. An extended round may be performed within the forward auction if the final stage rule is not met but bidding stops in high-demand markets. Figure 2 illustrates the integration of the two auctions.

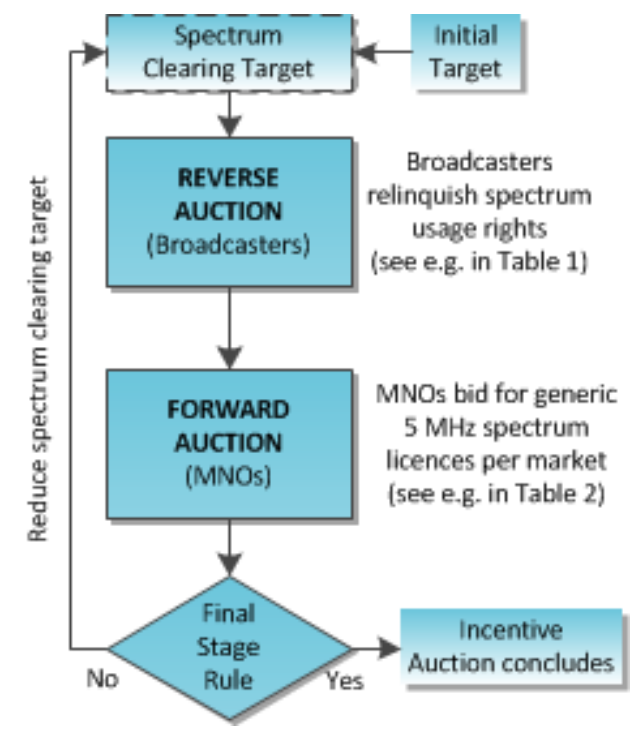

Figure 2: Integration of the reverse and forward auctions. 
The opening prices are the starting prices for the reverse auction, and they are the highest prices offered to TV stations for the three options to relinquish UHF spectrum usage rights: go off the air, move to a low VHF channel or move to a high VHF channel. The opening prices will be published by the FCC before the auction, and it is expected that they will be very high in order to gather interest from broadcasters. Prices will be calculated systematically for each station taking into account its covered population and the potential interference in the repacking process, and a uniform price (base clock price) for a UHF station going off the air. For moving to low VHF and high VHF, tentative price ranges are between 67 and 80 percent, and between 33 and 50 percent of the station's price to go off-air, respectively.

The initial spectrum clearing target for the first round will be determined based on the initial position of the broadcasters indicated in the pre-auction application process. The reverse auction will start at highest clearing target possible based on the eleven potential scenarios for the 600 $\mathrm{MHz}$ band plan described in Section III.

The first reverse auction bidding process will determine the total amount of incentive payments to broadcasters required to clear the initial clearing target. If the proceeds of the forward auction cover the bids of the reverse auction, the TV broadcaster relocation fund (USD \$1.75 billion) [3], the auction costs of the FCC, and the average price per MHz-POP ${ }^{1}$ satisfies the minimum requirement established by the FCC before the auction, then the incentive auction will close at the initial spectrum clearing target. If not, additional rounds will be run with progressively lower spectrum targets in the reverse auction, and consequently less spectrum available in the forward auction, until the final stage rule is satisfied. An extended round may be performed within the forward auction if the final stage rule is not met but bidding stops in high-demand markets in order to give MNOs the opportunity to express demand at higher prices to meet the final stage rule, avoiding the need to move to a lower clearing target.

\subsection{The Reverse Auction}

The reverse auction will follow a descending clock auction format, in which prices are progressively reduced until the number of RF channels that broadcasters are willing to move or relinquish matches the clearing target. In each bidding round, broadcasters will be offered prices for one or more bid options, and they will have to indicate their choices at those prices. The descending clock structure allows broadcasters to react to the prices provided by the reverse auction, rather than having to formulate their own bids.

Table 1 illustrates an example of the reverse auction with a clearing target of two stations in a given market. The opening price is $\$ \$ \$$, and 5 broadcasters (stations) accept this price. For the sake of simplicity, all stations are assumed technically identical. Since there are more stations than required (i.e., excess of demand), a new round of bidding is carried out with a lower bid price (in the example, $\$ \$$ ). Only three broadcasters accept this price, but since there is 1 station above the clearing target, another round is performed with a lower price of $\$$. Only one broadcaster accepts this price, and hence the two broadcasters which accepted a price of $\$ \$ \$$ will have to indicate the price level between $\$ \$$ and $\$ \$ \$$ (i.e., between the opening and closing prices of a round) which they accept to relinquish their station. This methodology is known as intra-round bidding, and it will allow broadcasters to drop out of the auction and remain on air if their target price is not satisfied.

\footnotetext{
${ }^{1} \mathrm{MHz}-\mathrm{POP}$ is calculated as follows: the total price paid for a license is divided by the product of the bandwidth in $\mathrm{MHz}$ times the population covered by the license; the FCC initially proposed an average price per MHz-POP benchmark of $\$ 1.25$ in the largest 40 markets by population [3].
} 


\begin{tabular}{|c|c|c|c|}
\hline Round & $\begin{array}{c}\text { Bid } \\
\text { Price }\end{array}$ & $\begin{array}{c}\text { Stations accepting } \\
\text { Bid Price }\end{array}$ & $\begin{array}{c}\text { Stations in excess } \\
\text { of Clearing Target }\end{array}$ \\
\hline I & $\$ \$ \$$ & $\$ \$$ & +3 \\
\hline II & $\$ \$ \$$ & -1 & +1 \\
\hline
\end{tabular}

Table 1: Illustrative reverse auction example where two stations are needed.

From the example, it can be noted that not all stations willing to accept an offered price will be selected to relinquish their licenses. Furthermore, stations in practice are not technically identical, as assumed in the example. This means that some stations are more suitable than others to fulfill a given spectrum clearing target, which also depends on the actual stations that participate in the auction. Hence, the reverse auction system will take into account the feasibility of repacking the remaining TV stations after the auction when offering the prices to each station. If a station cannot be assigned an RF channel, its price will become frozen, and will not be adjusted downwards in the subsequent rounds. Otherwise, stations will continue to be offered reduced prices as long as they become necessary to meet the spectrum clearing target.

\subsection{The Forward Auction}

The forward auction will come after the reverse auction with an ascending clock auction format, in which prices are progressively increased until the number of spectrum licenses demanded by the MNOs matches the available spectrum (i.e., prices start low and are adjusted upwards). In short, prices will continue to rise until there is no excess demand for the available spectrum. Another important feature of the forward auction is that MNOs will bid on the desired number of generic paired $5 \mathrm{MHz}$ spectrum blocks per market (i.e., a $5 \mathrm{MHz}$ block for the uplink and another $5 \mathrm{MHz}$ block for the downlink). After the auction, a separate auction round process will be carried to allocate the specific blocks per market to the MNOs.

Table 2 illustrates an example of the forward auction for three available spectrum blocks in a given market in which only two MNOs are interested to obtain new licenses. The opening bid price is $\$$, and for this price the two MNOs demand three spectrum blocks each, hence having an excess demand of three blocks. Another round is carried out with a higher price per block $(\$ \$)$, which reduces the demand of both operators to two blocks. The third round increases the bid price to $\$ \$ \$$, which reduces the demand of the two MNOs to one block each. The price for the third block would be the highest bid of the two MNOs between $\$$ and $\$ \$$ (i.e., the opening and closing prices of the round). That is, the same intra-round bidding scheme used in the reverse auction can be applied in the forward auction.

\begin{tabular}{|c|c|c|c|c|}
\hline Round & $\begin{array}{c}\text { Price per } \\
\text { Spectrum Block }\end{array}$ & $\begin{array}{l}\text { Demand } \\
\text { MNO1 }\end{array}$ & $\begin{array}{l}\text { Demand } \\
\text { MNO2 }\end{array}$ & $\begin{array}{l}\text { Excess } \\
\text { Demand }\end{array}$ \\
\hline III & $\$ \$ \$$ & 事 & 1 & -1 \\
\hline II & $\$ \$$ & 14 & 1 & +1 \\
\hline I & $\$$ & 14 & 事 & +3 \\
\hline
\end{tabular}


Table 2: Illustrative forward auction example where two spectrum blocks (licenses) are available.

\subsection{TV Broadcast Spectrum Repacking}

Terrestrial broadcast TV in the U.S. employs $222 \mathrm{MHz}$ of spectrum in the UHF band and 72 $\mathrm{MHz}$ in the VHF bands, with a total number of over eight thousand TV stations. The frequency planning for the first-generation U.S. DTT standard ATSC was based on a multi-frequency network topology, where each TV station is assigned a $6 \mathrm{MHz} \mathrm{RF}$ channel to cover a particular geographical area. In the U.S., TV stations are classified as full power ("Full Power"), class A low power television ("Class A"), low-power television ("LPTV"), or TV translator ("Translator"). Full-power stations are traditional high-power high-tower stations with up to 1 MW effective radiated power (ERP) in the UHF band, and have the highest priority regarding RF interference. LPTV stations are limited up to $1 \mathrm{~kW}$ ERP in the UHF band, and also cover translators which retransmit the signals of a TV broadcast station. LPTV stations and translators are considered secondary to full-power stations, unless they are classified as class A. According to FCC, there are 1782 full power stations, 465 class A low power stations, 1980 LPTV, and 4175 translators. It should be pointed out that only full-power (commercial and noncommercial) and class A licensees are eligible to participate in the incentive auction, and that licensees of LPTV and TV translator stations cannot participate.

As part of the reverse auction, the TV stations that remain on air would be reorganized so that they occupy a smaller portion of the UHF band toward the low frequency side allowing repurposing a contiguous portion of the high frequency side of the UHF band for mobile use. The terms of the repacking process have been designed to preserve the population and coverage served by each of the remaining individual TV stations as was determined for a baseline back in February 2012. A new RF channel assignment will not be allowed if the population served is reduced by more than $0.5 \%$ due to interference. The FCC is planning to use a software tool to determine repack feasibility. This tool makes use of two files. One file contains possible repack channels for each station that are based on restrictions associated with interference protection into Canada and Mexico and with protection of public safety allocations. The second file contains the channels for each station and for each of these channels the stations which would cause the co and adjacent interference over the $0.5 \%$ limit.

\section{The 600 MHz Band Plan}

The TV spectrum incentive auction requires a flexible band plan for the $600 \mathrm{MHz}$ band because the quantity of broadcast spectrum which will be repurposed for mobile broadband will be an outcome of the auction itself. In this section we explain the flexible framework to account for different amounts of cleared spectrum. Ideally, after the reverse auction the same amount of spectrum will be available nationwide. The possibility of modifying the framework to accommodate varying amounts of available mobile spectrum per market is currently one of the open topics under discussion. Doing so would result in impaired spectrum blocks and a reduction in the number of available spectrum blocks in some markets. The FCC has initially proposed two categories of generic spectrum blocks: category 1 with at most $15 \%$ of the population impaired by interference, and category 2 with over $15 \%$ up to $50 \%$ of interfered population (spectrum blocks with more than $50 \%$ of impaired population will not be offered in the auction), having different price clocks for each category, and reducing the prices of the impaired blocks proportionally to the percentage of interfered population [3]. The final stage rule would only apply to category 1 blocks in the most populated markets, and hence after the final stage rule is satisfied the forward auction will continue until there is no excess demand in any category in any market. 
Figure 3 shows the eleven potential scenarios that have been defined depending on the amount of spectrum cleared in the auction, ranging from a maximum of $144 \mathrm{MHz}$ (24 RF channels) down to a minimum of $42 \mathrm{MHz}$ (7 RF channels). The amount of spectrum reallocated to mobile services will range between $120 \mathrm{MHz}$ in the most aggressive scenario and only $20 \mathrm{MHz}$ in the most conservative scenario. The envisaged frequency plan for mobile services consists of a frequency division duplexing (FDD) scheme with specific paired uplink and downlink bands comprised of $5 \mathrm{MHz}$ frequency blocks. The number of RF channels in the UHF band available for the repacking of broadcast TV licenses that remain on air would be reduced from 37 (from 14 up to 51, except RF channel 37, which is allocated to radio astronomy, RAS, and wireless medical telemetry services, WMTS) down to 12 in the most aggressive scenario (30 in the most conservative scenario). The FCC has initially proposed $70 \mathrm{MHz}$ as forward auction spectrum benchmark, corresponding to a broadcast spectrum clearing target of $84 \mathrm{MHz}$ [3]. It should be pointed out that the RF channel 37 will continue being reserved for RAS and WMTS applications after the incentive auction. In the figure, it can also be noted that each scenario includes guard bands between the different technologies, including a duplex guard band of 11 $\mathrm{MHz}$ between the uplink and the downlink mobile transmissions. The guard band between broadcast and mobile services would range between 7 up to $11 \mathrm{MHz}$, which is intended to avoid interference issues in adjacent channels [4].

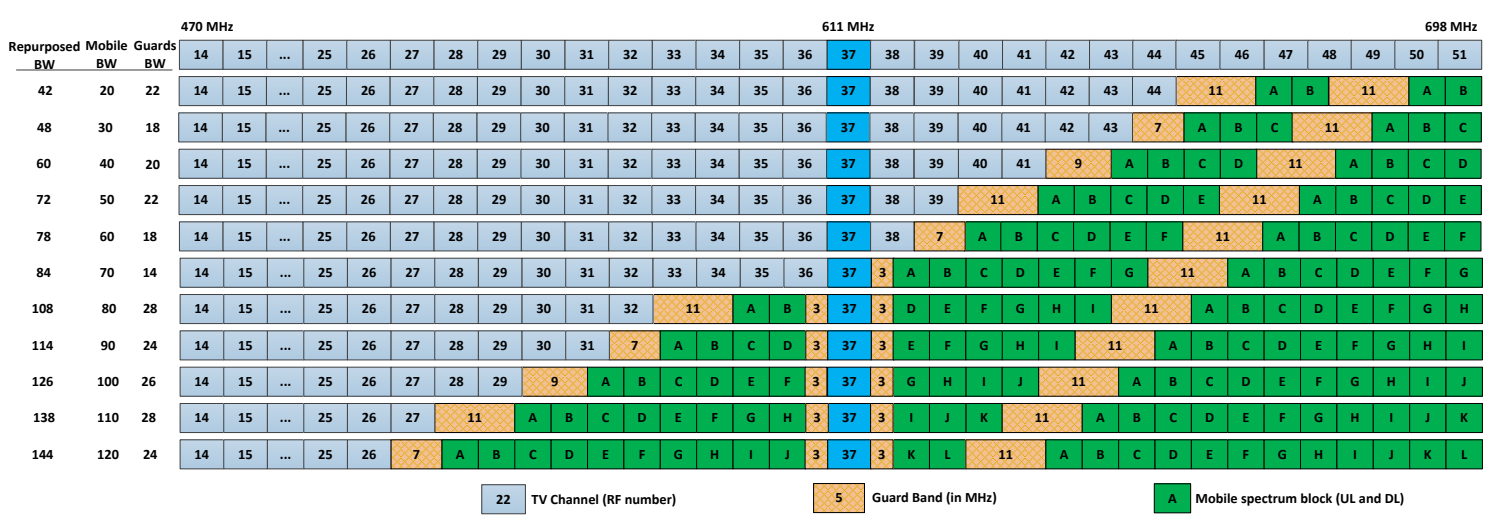

Figure 3: Potential scenarios for the $600 \mathrm{MHz}$ band plan after the incentive auction.

In the U.S., unlicensed devices can operate on broadcast television RF channels that are not used at a given location, known as TV white spaces. Unlicensed devices are to use certified radio equipment, may not cause harmful interference to licensed incumbent services, and must accept any interference that they receive. Interference from TV white space devices into the incumbent services is supposed to be controlled through a spectrum database of protected incumbent service areas. With the new $600 \mathrm{MHz}$ band, unlicensed devices and wireless microphones would be allowed to operate in TV white spaces in the repacked broadcast TV band, but also in the new guard bands of the resulting $600 \mathrm{MHz}$ band plan. Furthermore, the FCC has proposed to allow unlicensed devices to operate for the first time on RF channel 37 by establishing interference protection margins for existing operations in the white space database. This proposal seeks to promote greater innovation in new products and services by enabling spectrum for unlicensed devices on a nationwide basis because currently the amount of available spectrum is very limited or even nonexistent in many major markets.

\section{Challenges of The TV Spectrum InCEntive AuCtion}

The TV spectrum incentive auction is a very challenging process because all the pieces have to work together in order to be successful. For example, the opening bids by the FCC could break the auction from the beginning, as they will directly influence the participation of the 
broadcasters and the levels at which they might potentially drop out of the process. Efficient repacking of the remaining $\mathrm{TV}$ stations that remain on air after the auction must also be accomplished as a component of a successful auction. The repacking algorithm may be the most complex technical element of the overall process, which will be used in the reverse auction to help determine if a bid of a TV station is critical to the relevant spectrum clearing target.

Migrating from the UHF band down to the VHF band (and from the high-VHF down the lowVHF) may imply reception issues for the viewers. Although signals would reach further, building penetration loss would increase and, in order for them to maintain their efficiency, the size of customer receive antennas would need to increase.

The incentive auction will greatly affect the use of wireless microphones, low power TV stations (not classified as class A), and unlicensed TV white spaces devices, since all their operation would have to be modified after the repacking process.

A main drawback of the incentive auction process is that broadcasters that desire to continue their businesses well into the future may not have enough spectrum available for introducing new technologies.

\section{ATSC 3.0}

From an alternate perspective, the incentive auction represents a tremendous opportunity for broadcasters who are interested in thriving in the business well into the future by embracing the technological advancements that are available and would greatly enhance the consumer experience. In comparison to the high costs of the repacking process ( $\$ 2$ million to $\$ 5$ million per station), which may not be fully covered by the broadcast relocation fund, the additional costs to migrate to a next generation system may not be significant ( $\$ 250$ thousand to $\$ 500$ thousand per station).

The Advanced Television Systems Committee (ATSC) is currently developing its nextgeneration TV broadcasting system, known as "ATSC 3.0," which aims to become the reference DTT technology worldwide, leveraging all the progress beyond prior state-of-the art. The ATSC 3.0 system is being designed to include, among other features, higher system capacity to deliver a combination of emerging Ultra-High Definition (UHD), High Frame Rate (HFR), and High Dynamic Range (HDR) services and robust indoor and mobile reception. A primary goal is to simultaneously reach both fixed and portable devices. It is expected that ATSC 3.0 will allow transmitting more than $30 \mathrm{Mb} / \mathrm{s}$ in a $6 \mathrm{MHz} \mathrm{RF}$ channel for the same operation point of ATSC $1.0(15 \mathrm{~dB}$ signal-to-noise ratio at $19.4 \mathrm{Mb} / \mathrm{s})$ [5]. Therefore, the combination of ATSC 3.0 with the new video codec HEVC (High-Efficiency Video Codec), which, theoretically, provides up to four times the compression gain with respect to MPEG-2 video coding (two times with respect to MPEG-4/AVC), and its one-to-everyone broadcast architecture results in the most spectrally efficient mass media content delivery system.

One of the problems with the introduction of ATSC 3.0 may be the lack of spectrum to allow for a simulcast period with both the existing service and the new service, to allow users to progressively update their receivers either with a new TV set or a new set-top-box. One possibility may be for two broadcasters to share their two RF channels, such that one RF channel is used for ATSC 1.0 (since this configuration may allow for the delivery of two lower quality High-Definition HD services), and the other for ATSC 3.0.

Regarding the channel sharing of the new ATSC 3.0 services, broadcasters may be initially more interested in delivering a reduced set of TV channels (e.g., one each assuming that two broadcasters pair up) with a very robust configuration (e.g., SNR in the order of 0 to $3 \mathrm{~dB}$ ), aiming for robust indoor reception on TVs and tablets. Another possibility would be to deliver 
two higher quality HD fixed services per RF channel. In both cases, ATSC 3.0 would enable the transmission of new services once the simulcast period is concluded and more spectrum becomes available.

\section{OUTLOOK}

The upcoming broadcast TV spectrum incentive auction in the U.S. will be a groundbreaking spectrum event worldwide. This innovative spectrum auction scheme is a market-driven tool for repurposing spectrum. With a successful incentive auction, the U.S. would be the first country to make the upper portion of the $600 \mathrm{MHz}$ band available for mobile broadband.

The incentive auction is a very challenging process from both technical and economic points of view because in order to be successful the reverse and forward auctions and the repacking process must work seamlessly. The opportunities that the repacking component of the auction presents to TV broadcasters should not be disregarded. The introduction of the ATSC 3.0 next generation digital terrestrial television system makes possible the delivery of higher quality services to an entirely new set of display devices and allows for the service to continue being a competitive media platform. Therefore, the migration to ATSC 3.0 should be taken into account as part of the overall incentive auction.

\section{REFERENCES}

[1] European Broadcasting Union (EBU), "Spectrum Fact Sheet," July 2014.

[2] P. Lamy, "Results of the work of the high level group on the future use of the UHF band (470-790 MHZ)," Report to the European Commission, Sept. 2014.

[3] Federal Communications Commission (FCC), "Learn Program about Incentive Auctions," FCC.gov/LEARN.

[4] M. Fuentes, C. García-Pardo, E. Garro, D. Gómez-Barquero, and N. Cardona, "Coexistence of digital terrestrial television and next generation cellular networks in the $700 \mathrm{MHz}$ Band," IEEE Wireless Comm. Mag., vol. 21, no. 6, Dec. 2014.

[5] L. Michael and D. Gómez-Barquero, "Modulation and Coding for ATSC 3.0," Proc. IEEE Broadband Multimedia Systems and Broadcasting, Ghent, Belgium, 2015.

\section{Author Biographies}

DAVID GÓMEZ-BARQUERO (dagobar@iteam.upv.es) received a Ph.D. degree in telecommunications engineering from the Universitat Politècnica de València (UPV), Spain, in 2009. He is currently a senior researcher (Ramon \& Cajal Fellow) at UPV's Institute of Telecommunications and Multimedia Applications (iTEAM), where he leads a research group working on the development of next-generation digital terrestrial broadcast technologies, and a guest research scholar at New Jersey Institute of Technology (NJIT). He is the editor of the book Next Generation Mobile Broadcasting (CRC Press, 2013), and the vice-chairman of the Modulation and Coding Ad-Hoc Group of the standardization process of the next-generation U.S. TV broadcasting system ATSC 3.0.

M. WINSTON CALDWELL, P.E. (winston.caldwell@fox.com) received his Bachelor of Engineering degree in electrical engineering from Vanderbilt University and his Master of Science degree in electrical engineering from the University of Southern California. He is a licensed Professional Engineer in the state of California with over twenty years of electrical engineering experience, specializing in RF propagation, wireless communications, and antenna design. He is currently Vice President, Spectrum Engineering and Advanced Engineering, for 21st Century Fox's Fox Networks Group, where he is involved with the exploration of new broadcasting opportunities including development work on the ATSC 3.0 next-generation TV 
broadcast standard, acting as vice-chairman of the Waveform Ad-Hoc Group. He is an active participant in the ITU, IEEE, NAB, NABA and SMPTE, where he has provided technical expertise in the determination of compatibility requirements between the established broadcasting services and unlicensed TV band, ultra-wideband, power-line transmission and IMT-Advanced devices. 